\title{
Utilidad de la terapia de rescate en la erradicación de Helicobacter pylori: estudio longitudinal retrospectivo
}

\author{
Usefulness of rescue therapy in Helicobacter pylori eradication: retrospective longitudinal study
}

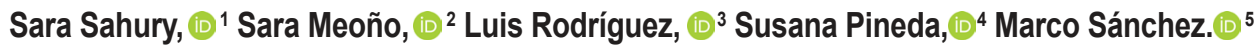

${ }^{1}$ Médica, Residente de Primer Año, Postgrado de Medicina Interna, Facultad de Ciencias Médicas (FCM), Universidad Nacional Autónoma de Honduras (UNAH). ${ }^{2}$ Médica General, egresada FCM UNAH, ${ }^{3}$ Residente de 3er Año del Postgrado de Medicina Interna, FCM UNAH, ${ }^{4}$ Médica Especialista en Gastroenterología, Servicio de Gastroenterología y Endoscopía, Departamento de Medicina Interna, Hospital Escuela Tegucigalpa, ${ }^{5}$ Médico Especialista en Gastroenterología, Departamento de Medicina Interna, Hospital Escuela, Tegucigalpa.

RESUMEN. Antecedentes: Las recomendaciones de esquemas para erradicar Helicobacter pylori se encuentran ampliamente disponibles. Este es un patógeno de alta prioridad para búsqueda y desarrollo de nuevos y efectivos tratamientos. Objetivo: Describir la respuesta terapéutica con terapia de rescate para infección por H. pylori, Hospital Escuela, Tegucigalpa, diciembre 2016-abril 2017. Métodos: Estudio descriptivo longitudinal retrospectivo en pacientes consecutivos con sintomatología gastrointestinal e infección confirmada por $H$. pylori. Mediante el registro del Servicio de Gastroenterología, Departamento de Medicina Interna, se identificaron pacientes positivos por $H$. pylori. Se registraron datos sociodemográficos, clínicos y diagnósticos. El tratamiento de rescate brindado fue, vía oral por 10 días: levofloxacina $500 \mathrm{mg} / \mathrm{dia}$, esomeprazol $40 \mathrm{mg}$ dos veces/día, amoxicilina $1 \mathrm{gr}$ dos veces/ día. La confirmación de la erradicación fue realizada 4-8 semanas postratamiento. Se registró información sobre la adherencia al tratamiento y los efectos secundarios. Resultados: Se analizaron 30 casos; $56.7 \%$ (17) pacientes nuevos y $43.3 \%$ (13) pacientes con al menos un fracaso. En el $16.0 \%$ (5) no hubo confirmación de erradicación; se obtuvo una tasa de erradicación del 72.0\% (18/25), IC95\% 50.6-87.9; siendo 78.5\% (11/14) en pacientes nuevos versus $63.6 \%$ (7/11) en fracasos previos, IC95\% -9.6-54.0, $p=0.318$. Discusión: La tasa de erradicación en este grupo de pacientes no fue satisfactoria. Actualmente el tratamiento con levofloxacina es recomendado como terapia de segunda línea o de rescate en regiones con baja o alta resistencia a la claritromicina, aunque la resistencia a quinolonas ha aumentado en los últimos años en varios países.

Palabras clave: Erradicación de la enfermedad, Fármacoresistencia Microbiana, Helicobacter pylori, Levofloxacina.

Recibido: 31-03-2020 Aceptado: 25-05-2021 Primera vez publicado en línea: 26-06-2021 Dirigir correspondencia a: Dra. Sara Sahury

Correo electrónico: danielasahury@gmail.com

Declaración de relaciones y actividades financieras y no financieras y conflictos de interés: ninguno.

DOI: https://doi.org/10.5377/rmh.v89i1.11642

(C) 2021 Autor(es): (c) (1)

\section{INTRODUCCIÓN}

Helicobacter pylori es una bacteria descrita morfológicamente como un bacilo Gram-negativo corto, espiral o en forma de $S .{ }^{1}$ Es un patógeno encontrado en los humanos, que causa gastritis crónica activa en todas las personas colonizadas por la bacteria. Esto puede progresar a úlceras, gastritis atrófica, adenocarcinoma gástrico y linfoma MALT (Tejido Linfoide Asociado a la Mucosa); la enfermedad causada por $H$. pylori es considerada como enfermedad infecciosa sin tomar en cuenta si el paciente esta sintomático o no, ni tampoco la etapa de la enfermedad. ${ }^{2}$ La transmisión ocurre principalmente personapersona, también por vía fecal-oral y oral-oral. El estado socioeconómico bajo, malas condiciones ambientales y vivir en hacinamiento se ha correlacionado con alta prevalencia. La prevalencia estimada es de casi $70 \%$ en países en desarrollo y se observa en mayor cantidad en la población joven. ${ }^{3}$

Existe indicación incuestionable para investigar y tratar la infección por $H$. pylori en todos los casos de úlcera duodenal y gástrica activas, tanto en las formas no complicadas, como en las complicadas (hemorragia, perforación y estenosis); se ha demostrado que la erradicación satisfactoria del microorganismo acelera la cicatrización de úlceras y disminuye la tasa de recidivas, así como las complicaciones. ${ }^{4}$ Los consensos y metanálisis publicados, así como la Agencia Internacional para la Investigación del Cáncer (IARC) recomiendan la erradicación global de la infección por $H$. pylori como medida de prevención primaria de cáncer gástrico, principalmente en áreas con elevada incidencia. ${ }^{5,6}$ En las últimas décadas han surgido recomendaciones para cambiar el enfoque en cuanto al manejo de $H$. pylori debido al incremento de la resistencia antibiótica. ${ }^{7}$

Se estipula que la combinación de antibióticos debe ser seleccionada de acuerdo con la resistencia identificada según área geográfica. En países latinoamericanos hay poca información actual respecto a la resistencia identificada, estudios recientes sugieren que la resistencia a la claritromicina puede estar al alza. Es cierto que también hay una tendencia al aumento en la resistencia a las quinolonas en Latinoamérica, esta tendencia aún no ha llegado al nivel de resistencia a los 
macrólidos, especialmente a la claritromicina. En consecuencia, los esquemas basados en quinolonas siguen representando un escenario prometedor en la erradicación de H. pylori. ${ }^{8}$ Se define una terapia de rescate como aquella que se utiliza en pacientes en los que ya se han sido tratados con la terapia tradicional (inhibidor de bomba de protones + amoxicilina + claritromicina) o la terapia cuádruple (incluye bismuto) y no ha habido éxito en la erradicación, sin embargo hay reportes en los que se indica puede utilizarse en pacientes nuevos que nunca han recibido ninguna terapia de erradicación en las zonas en las que se presenta resistencia a la claritromicina. ${ }^{2}$

Honduras cuenta con estudios analíticos y comparativos sobre los esquemas de tratamiento para $H$. pylori realizados en su mayoría en el occidente del país, ${ }^{9,10}$ obteniendo resultados satisfactorios. Actualmente se considera que el área geográfica es de gran influencia para asegurar la correcta toma de decisiones al prescribir un tratamiento adecuado., ${ }^{9,10}$ Fundamentado en lo anterior, este estudio se realizó con el objetivo de describir la respuesta terapéutica con terapia de rescate utilizando levofloxacina, esomeprazol y amoxicilina, en pacientes diagnosticados con infección por $H$. pylori y atendidos en la consulta externa del Servicio de Gastroenterología, Departamento de Medicina Interna, Hospital Escuela, en el periodo de diciembre 2016 a abril 2017.

\section{MATERIALES Y MÉTODOS}

Estudio descriptivo longitudinal retrospectivo en pacientes mayores de 18 años que asistieron a la Consulta Externa del Servicio de Gastroenterología, Departamento de Medicina Interna del Hospital Escuela, Tegucigalpa, con sintomatología gastrointestinal que orientaba a infección por $H$. pylori. Se revi- só el registro de pacientes para identificar los expedientes clínicos de los casos confirmados en el período de diciembre de 2016 a abril de 2017. En la Figura 1 se presenta un diagrama de flujo sobre el proceso de identificación de los casos.

Para cada caso se registraron datos sociodemográficos, antecedentes personales no patológicos, sintomatología, comorbilidades, antecedente de infección y resultado de antígeno (Ag) fecal y/o biopsia vía endoscópica. Se registró información sobre dos evaluaciones: la evaluación inicial al momento del diagnóstico y la segunda evaluación a los 3 meses registrando los efectos adversos al tratamiento, abandono del tratamiento 0 adherencia al mismo y la realización del examen confirmatorio de erradicación mediante las pruebas de Ag fecal y/o biopsia por vía endoscópica. Se consideró una respuesta terapéutica adecuada a la terapia de rescate a todo aquel paciente que después de haber tomado el tratamiento se le realizó una prueba de Antígeno fecal o una biopsia endoscópica con resultado negativo para el microorganismo en el tiempo estipulado de la medición.

Los pacientes recibieron tratamiento de rescate para erradicar la bacteria utilizando levofloxacina $500 \mathrm{mg}$ v.o. una vez al día, esomeprazol $40 \mathrm{mg}$ v.o. dos veces al día y amoxicilina $1 \mathrm{~g}$ v.o. dos veces al día, con una duración de 10 días. Se realizó una comparación entre los grupos de pacientes nuevos y pacientes con fracaso previo. Adicionalmente se caracterizaron los pacientes que no lograron la erradicación. La tasa de erradicación se calculó realizando una sumatoria entre los pacientes que resultaron negativos mediante la prueba de antígeno fecal o por medio del resultado de la biopsia vía endoscópica dividida entre el total de pacientes que recibieron tratamiento y se realizaron su prueba confirmatoria de erradicación. Los datos recopilados fueron digitados y procesados en una base de datos electrónica diseñada con el paquete estadístico Epi

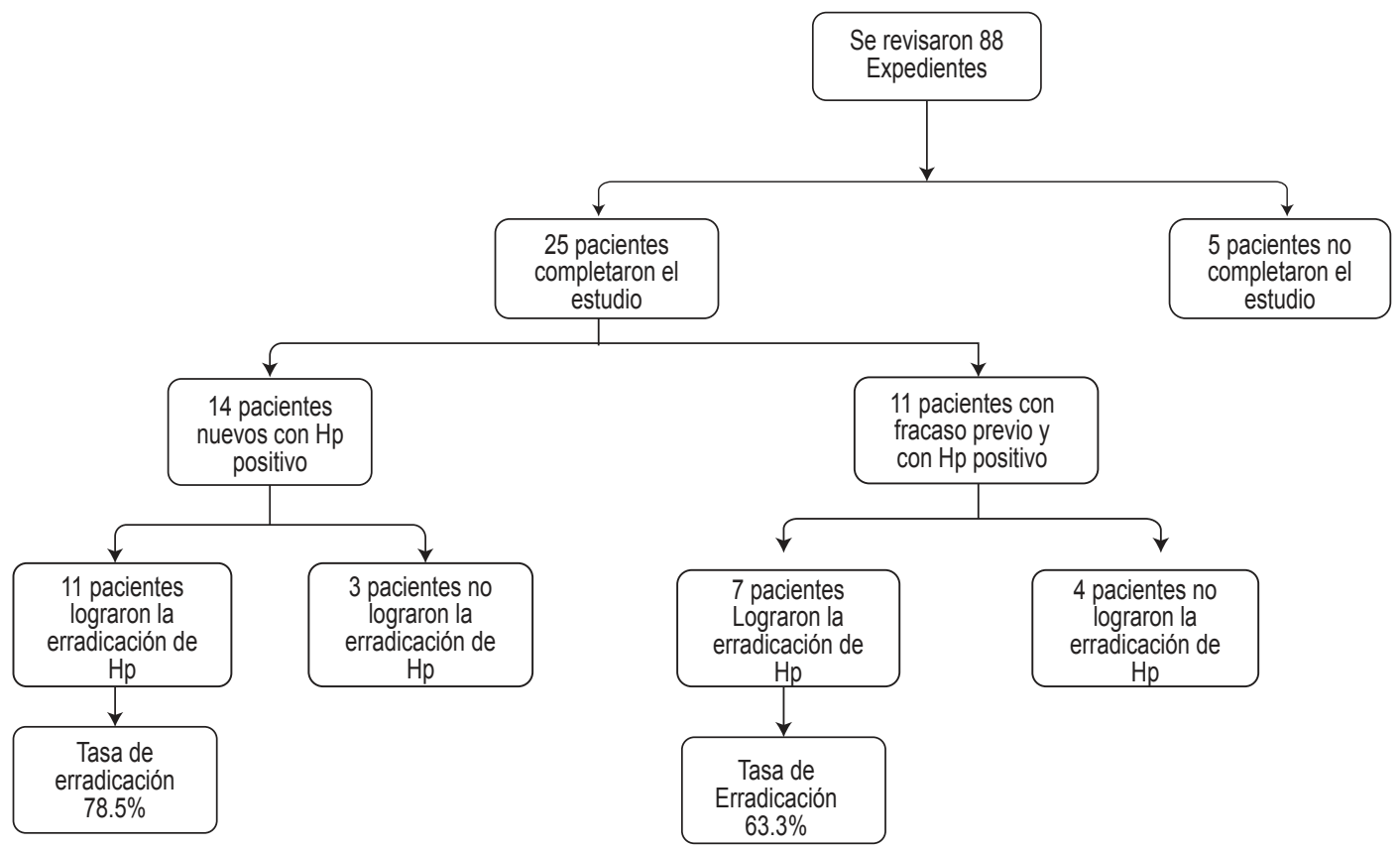

Figura 1. Diagrama de flujo de los participantes, utilidad de la terapia de rescate en la erradicación de Helicobacter pylori, Hospital Escuela, $2016-2017$. $\mathrm{Hp}=$ Helicobacter pylori. 
Info 7 versión 7.2.2.6 (para Windows). Se aplicaron frecuencias, porcentajes y se realizaron diferencia de proporciones para las variables cualitativas con el método de Fisher en el programa estadístico Minitab, determinándose intervalos de confianza del 95\% para la significancia estadística entre los grupos de pacientes que lograron la erradicación y los que no lo lograron. La información personal de los participantes fue manejada con confidencialidad.

\section{RESULTADOS}

Se revisaron 88 expedientes clínicos del período diciembre 2016 a abril 2017, identificando una muestra de 30 pacientes positivos para H. pylori (Ver Figura 1). De estos 30 casos, el $96.6 \%$ (29) fue diagnosticado con presencia del microorganismo mediante biopsia vía endoscópica y 3.3\% (1) resultó positivo mediante la prueba de antígeno fecal. El 90.0\% (27) presentó un resultado histológico de gastritis crónica. Entre las características sociodemográficas identificadas se encontró una edad media de 49.3 años, el sexo predominante fue el femenino con un $63.3 \%$ (19). El 83.2\% (25) residía en el municipio del Distrito Central (Ver Cuadro 1).

El $83.3 \%(25)$ regresó a su cita de confirmación de erradicación, de los cuales $56.0 \%$ (14) eran pacientes nuevos y $44.0 \%$ (11) pacientes con fracaso previo (ver Cuadro 1). De estos 25 casos, en la primera etapa del estudio se identificaron los ante-

Cuadro 1. Características epidemiológicas de los pacientes positivos para Helicobacter pylori, Hospital Escuela 2016-2017, $n=25$.

\begin{tabular}{lr}
\hline Datos sociodemográficos y clínicos & $\mathrm{N}(\%)$ \\
\hline Edad & \\
$20-39$ años & $11(44.0)$ \\
$40+$ & $14(56)$ \\
Media=49.3 & \\
Sexo & \\
Femenino & $19(76.0)$ \\
Masculino & $6(24.0)$ \\
Procedencia & \\
Zona rural & $3(12.0)$ \\
Zona urbana & $22(88.0)$ \\
Consumo crónico de AINES & \\
No & $15(60.0)$ \\
Sí & $10(40.0)$ \\
Alcoholismo & \\
No & $20(80.0)$ \\
Sí & $5(20.0)$ \\
Tabaquismo & \\
No & $22(88.0)$ \\
Sí & $3(12.0)$ \\
Sintomatología & \\
Pirosis & $19(76.0)$ \\
Dolor abdominal & $18(72.0)$ \\
Regurgitación & $16(76.7)$ \\
Dispepsia & $11(64.0)$ \\
Reflujo & $10(40.0)$ \\
Halitosis & $8(32.0)$ \\
Melena & $6(24.0)$ \\
Hematemesis & $5(20.0)$ \\
\hline
\end{tabular}

cedentes relacionados a enfermedad gástrica; el consumo crónico de antiinflamatorios no esteroideos (AINEs) estaba presente en $40 \%$ (10); alcoholismo fue referido en $20 \%$ (5). El antecedente de tabaquismo se presentó en $12 \%$ (3). No encontrándose en los pacientes con fracaso previo. Los síntomas más frecuentes al momento de presentarse a consulta fueron pirosis $76.0 \%$ (19) y dolor abdominal $72.0 \%$ (18), el promedio de duración de los síntomas fue de 4.6 meses en los pacientes nuevos y 9 meses en los pacientes con fracaso previo (Cuadro 1). El 48.0\% (12) tenía una o más comorbilidades, siendo hipertensión arterial la que se presentó con mayor frecuencia en ambos grupos, nuevos $28.0 \%(4 / 14)$ vs fracaso previo $27.2 \%(3 / 11)$.

La tasa de erradicación de $H$. pylori con el tratamiento de rescate fue calculada con los pacientes que asistieron a su cita de seguimiento que representaron un total de $83.3 \%$ (25/30), logrando que un $72.0 \%$ (18/25) (IC95\% 50.6-87.9) erradicara la bacteria con el tratamiento de rescate. Al comparar los grupos de pacientes nuevos y fracaso previo, se encontró que el $78.5 \%$ (11/14) de los pacientes nuevos y $63.6 \%$ (7/11) de los pacientes con fracaso previo lograron la erradicación (IC95\% $-9.6-54.0, p=0.318)$. El 28.0\% (7/25) no logró la erradicación, siendo un $21.4 \%$ (3/14) pacientes nuevos y en $36.3 \%$ (4/11) fracasos previos (IC95\% -66.1 - 37.5, $p=1.000$ ) (ver Figura 2). En los pacientes que erradicaron $H$. pylori, se encontró una media de 49.7 años; el $33.3 \%(6 / 18)$ eran hombres y el $66.7 \%(12 / 18)$ eran mujeres (IC-64.1-2.5, $p=0.094)$ ).

El $88.9 \%(16 / 18)$ de los pacientes curados provenía del área urbana y el $11.1 \%$ (2/18) del área rural (IC95\% 57.2 $98.3 p=0.000)$. Las enfermedades crónicas de base se encontraban presentes en un $52.0 \%$ (13/25), de los cuales el $69.2 \%$ (9/13) completó el estudio, presentándose la curación en $33.3 \%(6 / 18)$ de los pacientes. Se logró la erradicación en el $64.2 \%(9 / 14)$ de los pacientes nuevos que tuvieron adherencia al tratamiento de rescate y en el $54.5 \%$ (6/11) de pacientes con fracaso previo respectivamente (IC95\% -28.9 - 48.4, $p=0.697)$. Sin embargo, en el $14.2 \%(2 / 14)$ de los pacientes nuevos que incumplieron una o varias tomas del tratamiento lograron la erradicación y en 9.0\% (1/11) de los fracasos previos sin adecuada adherencia también lograron erradicar la bacteria (Cuadro 2).

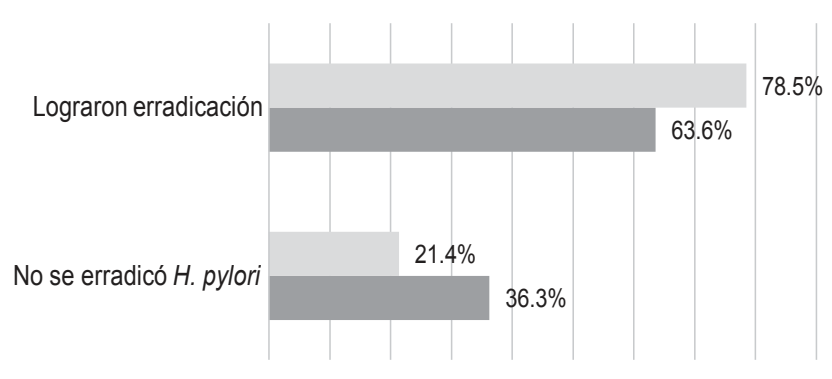

$0.0 \% 10.0 \% 20.0 \% 30.0 \% 40.0 \% 50.0 \% 60.0 \% 70.0 \% 80.0 \% 90.0 \%$

$$
\text { Pacientes nuevos Fracaso previo }
$$

Figura 2. Tasa de erradicación de Helicobacter pylori en pacientes nuevos y fracasos previos, utilidad de la terapia de rescate en la erradicación de Helicobacter pylori, Hospital Escuela, 2016-2017. 
Cuadro 2. Distribución de la erradicación de Helicobacter Pylori según características sociodemográficas, comorbilidades y adherencia al tratamiento de rescate, Hospital Escuela, 2016-2017, n=25.

\begin{tabular}{|c|c|c|c|c|}
\hline Características & $\begin{array}{c}\text { Paciente nuevo } \\
n=14 \\
N(\%)\end{array}$ & $\begin{array}{c}\text { Paciente con fracaso } \\
\text { previo } \\
n=11 \\
N(\%)\end{array}$ & Valor de $p$ & IC $95 \%$ \\
\hline $\begin{array}{l}\text { Erradicación } \\
\text { Sí } \\
\text { No }\end{array}$ & $\begin{array}{c}11(78.5) \\
3(21.4)\end{array}$ & $\begin{array}{l}7(63.6) \\
4(36.3)\end{array}$ & 0.656 & $-20.7-50.5$ \\
\hline \multicolumn{5}{|l|}{ Edad } \\
\hline $20-39$ & $4(28.5)$ & $2(18.1)$ & 1.000 & $-36.6-34.0$ \\
\hline $40+$ & $6(42.8)$ & $6(54.5)$ & & \\
\hline Promedio de edad & 47.5 & 50 & & \\
\hline \multicolumn{5}{|l|}{ Sexo } \\
\hline Masculino & $4(28.5)$ & $2(18.1)$ & 0.661 & $-22.4-43.2$ \\
\hline Femenino & $7(50.0)$ & $5(45.4)$ & 1.000 & $-34.8-43.9$ \\
\hline \multicolumn{5}{|c|}{ Lugar de residencia } \\
\hline Rural & $1(7.1)$ & $1(9.0)$ & 1.000 & $-23.6-19.7$ \\
\hline Urbana & $8(57.1)$ & $8(72.7)$ & 0.637 & $-52.5-21.3$ \\
\hline \multicolumn{5}{|l|}{ Con comorbilidad } \\
\hline Sí & $4(28.5)$ & $2(18.1)$ & 0.661 & $-22.4-43.2$ \\
\hline No & $1(7.1)$ & $2(18.1)$ & & \\
\hline \multicolumn{5}{|l|}{ Adherencia } \\
\hline Sí & $9(64.2)$ & $6(54.5)$ & 0.697 & $-28.9-48.4$ \\
\hline No & $2(14.2)$ & $1(9.0)$ & & \\
\hline
\end{tabular}

De los pacientes en los que se reportó consumo crónico de AINEs, lograron erradicar la bacteria en un $33.3 \%(6 / 18)$. Los pacientes con alcoholismo lograron un $11.1 \%(2 / 18)$ de la erradicación. Los pacientes con tabaquismo eran todos pacientes nuevos que recibían terapia de rescate; de los cuales $5.5 \%$ (1/18) erradicó la bacteria. Los pacientes que no lograron la erradicación fue del $28.0 \%$ (7/25). Las características de los pacientes en los que no se logró la erradicación tuvieron un promedio de edad de 42.4 años, el 100.0\% (7/7) eran mujeres, siendo $21.4 \%$ (3/14) pacientes nuevos y $36.3 \%$ (4/11) pacientes con fracaso previo, todas provenían del área urbana y $42.8 \%$ (3/7) presentaban comorbilidades (hipertensión arterial, asma, diabetes e hipotiroidismo); un 14.2\% (1/7) tenía antecedente de alcoholismo. El 71.4\% (5/7) tuvieron adherencia completa al tratamiento y $28.5 \%(2 / 7)$ incumplieron alguna vez su tratamiento, siendo las que incumplieron pacientes con fracaso previo. El efecto secundario al tratamiento brindado que notificaron con mayor frecuencia fue el sabor amargo en la boca en un 56.0\% (14/25) seguido por dolor abdominal el $44.0 \%$ (11/25) y náuseas el $40.0 \%(10 / 25)$.

Para la verificación de la erradicación el 84.0\% (21/25) se realizó biopsia vía endoscópica y el 16.0\% (4/25) por antígeno fecal; de los cuales el $64.0 \%$ (16/25) resultó negativo confirmado mediante biopsia vía endoscópica y el $8.0 \%(2 / 25)$ resultaron negativos con la prueba de antígeno fecal; en los pacientes que no se erradicó la bacteria el resultado de su confirmación fue verificado en un $20.0 \%(5 / 25)$ mediante biopsia y $8.0 \%$ (2/25) mediante antígeno fecal. El tiempo estimado de erradicación fue de aproximadamente 30 días.

\section{DISCUSIÓN}

En este estudio se encontró que la respuesta terapéutica no fue satisfactoria ya que la tasa de erradicación fue un $72.0 \%$. Entre las recomendaciones de la IV Conferencia Española de Consenso sobre el tratamiento de la infección por $H$. pylori, un tratamiento debe ser considerado efectivo cuando sea capaz de erradicar la infección por $H$. pylori en aproximadamente el $90 \%$ de los pacientes. ${ }^{11}$

En este estudio se efectuó una comparación entre los pacientes nuevos y los pacientes con fracaso(s) previo(s) en los que se erradicó y en los que no se erradicó la bacteria, encontrando bajos porcentajes de éxito en ambos grupos. El consenso de Maastricht $\checkmark$ realizado en la ciudad de Florencia refiere que luego del fracaso de un tratamiento, bien triple o cuádruple, que incluya claritromicina, se recomienda una pauta con levofloxacino, preferiblemente cuádruple (Inhibidor de bomba de protones (IBP), amoxicilina, levofloxacino y bismuto), o como alternativa, una terapia cuádruple con bismuto (IBP, bismuto, tetraciclina y metronidazol). ${ }^{11}$ En varios estudios se ha reportado la efectividad de la levofloxacina, amoxicilina y esomeprazol como tratamiento de primea línea o como terapia de rescate; en este estudio se dio tratamiento tanto a pacientes nuevos como a fracasos previos presentándose mayor porcentaje de erradicación en los pacientes nuevos; se han encontrado análisis que demuestran que la eficacia de la triple terapia con levofloxacina es superior al $90 \%{ }^{12,13}$

Se observaron diferentes circunstancias en los pacientes que no lograron la erradicación como el alcoholismo, la falta de adherencia al tratamiento, la mayor duración de la sintomatología y la presencia de comorbilidades, resultados que podrían influir y caracterizar el fracaso del tratamiento de rescate. Actualmente es recomendado como terapia de segunda línea o de rescate en regiones con baja o alta resistencia a la claritromicina; ${ }^{12,13}$ Aunque la resistencia a levofloxacina ha aumentado en los últimos años en varios países. Estudios más recientes demuestran que su eficacia va de $70 \%$ a $90 \%$, como en este estudio; en estos resultados se demuestra que la terapia ya no es tan efectiva como se había reportado anteriormente. ${ }^{14}$ Se conoce 
que la dificultad para erradicar $H$. pylori está relacionada con varias circunstancias, como múltiples sitios de crecimiento de la bacteria, resistencia a los antibióticos y sub-dosificación, falta de adherencia al tratamiento e inadecuada supresión de ácido.,15

En general los confirmados por $\mathrm{H}$. pylori eran mayores de 50 años y fue en este grupo precisamente donde se mostró mayor porcentaje de erradicación. Estudios realizados en países en vías de desarrollo han señalado que la infección por $H$. pylori se presenta principalmente en la población joven y que el contacto ocurre usualmente durante la primera década de la vida; hay reportes en los que más del $30 \%$ de las personas con la infección la han adquirido durante su juventud. Sin embargo, se ha observado que el estado seropositivo aumenta con la edad. ${ }^{3}$

Estudios internacionales de prevalencia muestran que existe un predominio del sexo masculino en cuanto a la infección por H. pylori. ${ }^{16,17}$ La región de Copán en Honduras fue parte de un ensayo clínico randomizado multicéntrico junto con otros 6 países en el año 2013, donde se encontró que no hubo diferencia en cuanto a la prevalencia del sexo, en este estudio hubo prevalencia del sexo femenino. ${ }^{18}$ Un punto importante que cabe mencionar en este estudio es la patología histológica que se reportó con mayor frecuencia en los hallazgos endoscópicos fue gastritis crónica al igual que en el estudio de Prevalencia de $H$. pylori en Enfermedad Acido-Péptica y Gastritis Crónica, y Respuesta Terapéutica realizado en 1997 en el Hospital Escuela. ${ }^{10}$

En este estudio se observó mayor población del área urbana. Varios estudios relacionan la resistencia antibiótica con la procedencia geográfica del paciente..$^{9,19-22}$ Es por eso, que los diferentes consensos sugieren firmemente que la elección del tratamiento se base en los patrones locales de resistencia, aunque el cultivo para $H$. pylori no siempre es realizado y en nuestro medio solo se realiza a nivel privado en raras ocasiones. En este estudio la población rural, aunque representada en minoría logró la erradicación, se cree que una buena respuesta terapéutica puede ser debida a menor exposición antibiótica según el estudio de Portillo $S$ y colaboradores, departamento de Ocotepeque, municipio La Encarnación, Honduras en el año 2019.22

Los pacientes con comorbilidades tuvieron buena adherencia al tratamiento, sin embargo, el $75.0 \%$ de los pacientes que no logró la erradicación tenía distintas comorbilidades que probablemente pudieron influir en el resultado final de su tratamiento. La presencia de comorbilidades no está establecida como modificador de la respuesta a los esquemas de erradicación de H. pylori, aunque es posible relacionar el riesgo de variabilidad en los niveles séricos de los fármacos por interacciones farmacológicas, y posiblemente un menor apego al esquema administrado a consecuencia del mayor número de medicamentos prescritos. $^{23}$

Los pacientes de este estudio tuvieron buena adherencia al tratamiento, destacando que los pacientes nuevos tuvieron mejor adherencia al tratamiento que los pacientes con fracaso(s) previo(s). La terapia fue bien tolerada por los pacientes, los efectos secundarios presentados durante el tratamiento fueron mínimos y leves, ninguno motivó la suspensión completa del mismo. La adherencia al tratamiento tiene un papel fundamental en la erradicación, aunque en la mayoría de los estudios no ha sido cuantificada. En ensayos clínicos se ha observado que la tasa de abandonos se relaciona con el número de dosis de medicamentos que toman los pacientes. La frecuencia y gravedad de los efectos adversos también son factores que influyen sobre la adherencia, sin embargo, se ha evidenciado que los pacientes cumplen mejor su tratamiento cuando conocen los efectos adversos potenciales y entienden en qué casos estaría justificado abandonar el tratamiento, por lo que es muy importante discutir los beneficios y riesgos del tratamiento. ${ }^{24}$

La búsqueda de atención médica en este estudio se basó en síntomas gastrointestinales característicos de úlcera péptica, se observó que la duración de los síntomas en los pacientes que no lograron la erradicación fue ligeramente mayor que en los pacientes que sí lograron la erradicación. En el estudio Cortes D, realizado en el Hospital Escuela, Honduras en 1997 sobre prevalencia de $\mathrm{H}$. pylori se reportó que el principal síntoma observado fue dolor en epigastrio; se destaca el hecho que es necesario realizar una historia y examen físico acuciosos. ${ }^{10,25,26}$

En este estudio se realizó biopsia vía endoscópica a más del $90 \%$ de los pacientes, por esto no podemos establecer una comparación de los resultados de ambos métodos. El miedo a la endoscopía ${ }^{27}$ en nuestra población y cultura todavía es muy arraigado lo que lleva a los pacientes a la elección de métodos no invasivos, como en este estudio en el que el $16.0 \%$ decidió verificar la erradicación mediante Ag fecal, único método disponible en el Hospital Escuela además de la biopsia vía endoscópica. A nivel privado estas pruebas tienen un costo elevado por lo que la población generalmente elige el que le resulte más cómodo económicamente. Existen métodos invasivos y no invasivos para diagnosticar infección por $\mathrm{H}$. pylori. ${ }^{15,25}$ Los resultados de varios estudios latinoamericanos concuerdan en que tanto los antígenos fecales como el test de aliento de la urea (UBT) son pruebas que aseguran la calidad en cuanto a sensibilidad y especificidad para verificar la erradicación de la infección como para el diagnóstico si no se requiere endoscopía digestiva alta, ya que, en caso contrario, la histología sigue siendo la mejor opción. . $^{15,28,29}$

En este estudio una minoría de pacientes presentó los antecedentes de riesgo establecidos para úlcera péptica, aun así, se observó que los pacientes con consumo crónico de AINEs lograron la erradicación. La infección por $H$. pylori y el consumo de AINEs, son los principales factores de riesgo para el desarrollo de complicaciones de la úlcera péptica, su erradicación mejora las tasas de curación y disminuye las complicaciones. ${ }^{30} \mathrm{Se}$ discute internacionalmente la asociación entre la infección por H. pylori y el consumo de alcohol y tabaco. En un estudio mexicano se llegó a la conclusión de que las personas con estos hábitos tenían mayor probabilidad de presentar la infección que las personas sin estos hábitos; ${ }^{31}$ algunos estudios muestran que los factores de riesgo asociados a gastropatías se encuentran implicados en la reducción de la eficacia de los tratamientos para la erradicación del $H$. pylori. ${ }^{32,33}$

La limitación del estudio fue el tamaño de la muestra, por lo que no podemos generalizar nuestros hallazgos al resto de los pacientes con $H$. pylori, ya que su dimensión no representa la realidad del hospital donde se realizó el estudio, también el 
tiempo de entrega del resultado de biopsia retrasa el diagnóstico y la confirmación de erradicación; el seguimiento se dificulta cuando los pacientes ya no presentan sintomatología alguna. El hospital no cuenta con todas las terapias para erradicación. En base a lo encontrado en la presente investigación se concluye que se deben realizar estudios en diferentes áreas geográficas del país, para establecer patrones locales de resistencia, incluyendo factores de riesgo, pacientes con comorbilidades, prevalencia por sexo, así como investigaciones de tipo cualitativo en busca de soluciones en cuanto a la adherencia al tratamiento. Por la complejidad de esquemas actuales, efectos adversos y evaluación del costo-beneficio de las intervenciones terapéuticas se recomienda que se tomen en cuenta todos estos aspectos para permitir una aproximación global en la evaluación del tratamiento ideal del H. pylori. ${ }^{34,35}$

\section{CONTRIBUCIONES}

Todos los autores participaron en la concepción, diseño del estudio, adquisición de la información bibliográfica, análisis e interpretación de datos y redacción final del manuscrito. SS, SM, LR, SP y MS, además contribuyeron en la recolección de los datos. Finalmente, todo el equipo de investigación atendió las recomendaciones editoriales y aprobaron la versión final del manuscrito.

\section{DETALLES DE AUTORES}

${ }^{1}$ Sara Sahury

${ }^{2}$ Sara Meoño

${ }^{3}$ Luis Rodríguez

${ }^{4}$ Susana Pineda

${ }^{5}$ Marco Sánchez danielasahury@gmail.com

sara.meono@gmail.com

luigicastellanos@yahoo.com

susana1375@yahoo.com.mx

doctor_sanchezhn@yahoo.com

\section{REFERENCIAS}

1. Macenlle R, Gayoso P, Sueiro R, Fernández J. Risk factors associated with Helicobacter pylori infection: A population-based study conducted in the province of Ourense. Rev Esp Enferm Dig. [Internet]. 2006[citado 21 mayo 2020];98(5):330-340. Disponible en: http://scielo.isciii.es/scielo. php?script=sci_arttext\&pid=S1130-01082006000500003\&lng=es.

2. Malfertheiner P, Megraud F, O'Morain C, Gisbert J, Kuipers E, Axon A, et al. Management of Helicobacter pylori infection the Maastricht V/Florence Consensus Report Gut 2017;66:6-30. DOI: 10.1136/gutjnl-2016-312288.

3. Palanduz A, Erdem L, Cetin BD, Ozcan NG. Helicobacter pylori infection in family members of patients with gastroduodenal symptoms. A crosssectional analytical study. Sao Paulo Med J. 2018;136(3):222-227. DOI: 10.1590/1516-3180.2017.0071311217.

4. Gisbert J. Infección por Helicobacter pylori; En: Montoro MA, García Pagán JC, editores. Gastroenterología y hepatología: problemas comunes en la práctica clínica 2a ed. España. Jarpyo Editores, 2012. p. 281-290.

5. Jiménez G. Helicobacter pylori como patógeno emergente en el ser humano. Rev Costarric Salud Pública [Internet]. 2018[citado 21 mayo 2020];27(1):65-78. Disponible en: http://www.scielo.sa.cr/scielo. php?script=sci_arttext\&pid=S1409-14292018000100065\&lng=en .

6. Portillo S. ¿Son aplicables los consensos Internacionales para le erradicación de H. Pylori a la realidad nuestra? Congreso Médico Nacional. San Pedro Sula, Cortés. 10-13 de julio 2019. Rev Med Hondur. 2019;87(1): S1-106.

7. Melese A, Genet C, Zeleke B, Andualem T. Helicobacter pylori infections in Ethiopia; prevalence and associated factors: a systematic review and meta-analysis. BMC Gastroenterology [Internet]. 2019[citado 21 mayo 2020];19:8. Disponible en: https://doi.org/10.1186/s12876-018-0927-3.

8. Ladrón de Guevara L, Bornstein Quevedo L, González Huezo S, Castañeda Romero B, Costa FG, di Silvio-López M. Erradicación de Helicobacter pylori en México con un esquema basado en levofloxacina versus la triple terapia estándar: resultados de un estudio clínico de fase iiib, abierto, aleatorizado, de no inferioridad. Rev Gastroenterol Mex. 2019; 84(3):274-283.

9. Portillo S, Suazo J, Hernández I, Chinchilla J, Lezama A, Zúñiga J, et al. Estudio comparativo entre terapia cuádruple con bismuto versus terapia concomitante para la erradicación de Helicobacter pylori en población hondureña mayor de 18 años que acude a consulta de gastroenterología; Centro de Enfermedades Digestivas, Hospital del Valle. Exposición de trabajos libres en cartel [Resumen]. Rev Gastroenterol Mex. 2018;83(2):100159.

10. Cortés D, Torres G. Prevalencia de Helicobacter pylori en enfermedad acido-peptica y gastritis crónica, y respuesta terapéutica. Rev Med Hondur [Internet]. 1997[citado 21 mayo 2020];65(4):101-106. Disponible en: http:// www.bvs.hn/RMH/pdf/1997/pdf/Vol65-4-1997-3.pdf

11. Gómez B, Castro L, Argüelles F, Castro C, Caunedo A, Romero M. Erradicación de Helicobacter pylori con terapia cuádruple con bismuto en pacientes naïve y fracasos previos: experiencia en la práctica clínica real. Rev Esp Enferm Dig [Internet]. 2017[citado 21 mayo
2020];109(8):552-558. Disponible en: http://scielo.isciii.es/scielo. php?script=sci_arttext\&pid=S1130-01082017000800003\&lng=es. http:// dx.doi.org/10.17235/reed.2017.4809/2016.

12. Xiao S, Gu M, Zhang G. Is levofloxacin-based triple therapy an alternative for first-line eradication of Helicobacter pylori? A systematic review and meta-analysis. Scand J Gastroenterol. 2014;49(5):528-538, DOI: 10.3109/00365521.2014.887765.

13. Alarcón J, Fernández G, Cortés E, Castañón C, Sampedro J, Cruz I, et al. Clarithromycin resistance and prevalence of Helicobacter pylori virulent genotypes in patients from Southern México with chronic gastritis. Infect Genet Evol [Internet], 2016[citado 14 abril 2020];44:190-198. Disponible en: http://www.sciencedirect.com/science/article/pii/S1567134816302684

14. Chen P, Wu M, Chen C, Bair M, Chou C, Lin J, et al. Systematic review with meta $\square$ analysis: the efficacy of levofloxacin triple therapy as the first $\square$ or second $\square$ line treatments of Helicobacter pylori infection. Aliment Pharmacol Ther. 2016; 44: 427-437. Doi:10.1111/apt.13712.

15. Otero R, Gómez Z, Otero P, Trespalacios R. Helicobacter pylori: ¿cómo se trata en el 2018? Rev gastroenterol Perú [Internet]. 2018[citado 12 abril 2020];38(1):54-63. Disponible en: http://www.scielo.org.pe/scielo. php?script=sci_arttext\&pid=S1022-51292018000100009\&Ing=es.

16. Correa S, Cardona A, Correa T, Correa L, García H, Estrada S. Prevalencia de Helicobacter pylori y características histopatológicas en biopsias gástricas de pacientes con síntomas dispépticos en un centro de referencia de Medellín, Hepatología. Rev Col Gastroenterol[Internet]. 2016[citado 12 abril 2020];31(1):9-15. Disponible en: http://www.scielo.org.co/pdf/rcg/ v31n1/v31n1a02.pdf

17. Peleteiro B, Bastos A, Ferro A, Lunet N. Prevalence of Helicobacter pylori infection worldwide: a systematic review of studies with national coverage. Dig Dis Sci [Internet]. 2014[citado 12 abril 2020];5(8):1698-709. Disponible en: https://www.ncbi.nlm.nih.gov/pubmed/24563236

18. Porras C, Nodora J, Sexton R, Ferreccio C, Jimenez S, Dominguez R, et al. Epidemiology of Helicobacter pylori infection in six Latin American countries (SWOG Trial S0701). Cancer Causes Control 2013; 24(2): 209-215.

19. Comisión económica para América Latina y el Caribe (CEPAL). Definición de población urbana y rural utilizadas en los censos de los países latinoamericanos. Censos de 1974 y 1988. [Internet]. Santiago: CEPAL; s.f. [citado 23 junio 2020] Disponible en: https://www.cepal.org/sites/default/ files/def_urbana_rural.pdf

20. Savoldi A, Carrara E, Graham D, Conti M, Tacconelli E. Prevalence of antibiotic resistance in Helicobacter pylori: a systematic review and metaanalysis in World Health Organization Regions. AGA journals. Gastroenterology [Internet]. 2018[citado 24 mayo 2020];155(5):1372-1382. Disponible en: https://www.gastrojournal.org/article/S0016-5085(18)34761-9/fulltext.

21. Otero W. Infección por Helicobacter pylori en 2019: ¿Cómo la debemos tratar en LATAM? En: VII Foro digestivo latinoamericano. Madrid: Ergon S. A; 2019. Módulo 1; 26-29.

22. Portillo S, Suazo J, Hernández I, Chinchilla J, Lezama A, López G. La te- 
rapia con claritromicina podría continuar siendo efectiva en la erradicación de Helicobacter pylori en la Encarnación, Ocotepeque. Rev Med Hondur [Internet]. 2019[citado 21 mayo 2020];87(supl 1): S1-106 Disponible en: http://www.bvs.hn/RMH/pdf/2019/pdf/Vol87-S1-2019.pdf

23. Gonzáles M, Rojas A, Rosales A, Cordero M, Hinojosa A, Mejía E, et al. Frecuencia de erradicación del Helicobacter pylori con triple esquema convencional en pacientes adultos del Centro Médico Issemym. Rev Gastroenterol Mex. 2012; 77 (3):114-118. DOI: 10.1016/j.rgmx.2012.05.001

24. Avalos R, Vanterpool M, Morales M, Lamoth I, Prendes A. Nuevos retos en el tratamiento de la infección por Helicobacter pylori. Rev Med Electrón [Internet]. 2019[citado 13 marzo 2020];41(4):979-992. Disponible en: http://scielo.sld.cu/scielo.php?script=sci_arttext\&pid=S168418242019000400979\&lng=es

25. Sáenz R. Helicobacter pylori, hoy: una historia de 30 años. Rev Méd Clín Las Condes. 2015; 26 (5): 572-578. DOI: 10.1016/j.rmclc.2015.09.004

26. Thiebaud L, Luque M, Sabillón L, Millares H, Bustillo K. Eficacia del tratamiento convencional para Helicobacter pylori en niños. Rev Med Hondur [Internet]. 2011[citado 12 abril 2020];79(2):65-67. Disponible en: http:// www.bvs.hn/RMH/pdf/2011/pdf/Vol79-2-2011-4.pdf

27. Wang MC, Zhang LY, Zhang YL, Zhang YW, Xu XD, Zhang YC. Effect of music in endoscopy procedures: systematic review and meta-analysis of randomized controlled trials. Pain Med. 2014;15(10):1786-94.

28. Nguyen T, Falcón R, Vázquez R, Almaguer T, Tamayo C, Corrales R, et al. Evaluación del desempeño de dos pruebas para la detección de antígeno de Helicobacter pylori en heces. Rev Cubana Med Trop [Internet]. 2017[citado 24 marzo 2020];69(1):1-7. Disponible en: http://scielo.sld.cu/scielo. php?script=sci_arttext\&pid=S0375-07602017000100006\&lng=es .

29. Reyes M, Trespalacios A, Otero W. Comparación de la prueba de antígenos fecales (Elisa) y test de aliento de la urea frente a histología para el diagnóstico de Helicobacter pylori: Revisión sistemática de la literatura. Rev Col Gastroenterol [Internet]. 2009[citado 24 marzo 2020];24(4):373381. Disponible en: http://www.scielo.org.co/pdf/rcg/v24n4/v24n4a07.pdf

30. García A, Vanterpool H, Morales M, Lamoth W, Huerta A. Nuevos retos en el tratamiento de la infección por Helicobacter pylori. Rev Méd Electrón [Internet]. 2019[citado 24 marzo 2020];41(4). Disponible en: http://www. revmedicaelectronica.sld.cu/index.php/rme/article/view/2983/4393

31. Sanchez J, Cabrales A, Magaña G, Peraza F. Infección por Helicobacter pylori y su asociación con el consumo de alcohol. Estudio de casos y controles. Rev Gastroenterol Mex [Internet]. 2013[citado 23 mayo 2020]; 78(3):144-150. Disponible en: https://doi.org/10.1016/j.rgmx.2013.06.003

32. Rodríguez J, Boffill A, Rodríguez L, Losada J, Socías Z. Factores de riesgo asociados a la gastritis aguda o crónica en adultos de un hospital ecuatoriano. MEDISAN [Internet]. 2019[citado 21 abril 2020];23(3). Disponible en: http://www.medisan.sld.cu/index.php/san/article/view/2457

33. Castellanos K, Lorenzo F, Cabrales J, Carpio E, Carrazana K. Factores de riesgo asociados con las gastropatías. Trinidad, Sancti Spíritus. Revista Finlay [Internet]. 2013[citado 17 marzo 2020]; 3(3). Disponible en: http:// revfinlay.sld.cu/index.php/finlay/article/view/198

34. Rollán A, Giancaspero R, Acevedo C, Fuster F, Hola K. Tratamiento de la infección por Helicobacter pylori en pacientes con úlcera duodenal: Estudio de costo-beneficio. Rev méd Chile [Internet]. 2000[citado 17 marzo 2020];128(4):367-377. Disponible en: https://scielo.conicyt.cl/scielo. php?script=sci_arttext\&pid=S0034-98872000000400002\&lng=es.

35. Sierra F, Forero J, Rey M. Tratamiento ideal del Helicobacter pylori: una revisión sistemática. Rev Gastroenterol Mex. 2014; 70(1): 28-49. DOI: 10.1016/j.rgmx.2013.03.003

ABSTRACT. Background: Scheme recommendations to eradicate Helicobacter pylori are widely available; this is a high priority pathogen for the search and development of new and effective treatments. Objective: To describe the therapeutic response of rescue therapy in $\mathrm{H}$. Pylori infection, Hospital Escuela, Tegucigalpa, december 2016-april 2017. Methods: Retrospective longitudinal descriptive study in consecutive patients with gastrointestinal symptoms and confirmed $H$. pylori infection. Through the registry of the Gastroenterology Service, Department of Internal Medicine, positive patients for $H$. pylori were identified. Sociodemographic, clinical and diagnostic data were registered. The rescue treatment provided was orally for 10 days: levofloxacin $500 \mathrm{mg} /$ day, esomeprazole $40 \mathrm{mg}$ twice / day, amoxicillin $1 \mathrm{~g}$ twice / day. Confirmation of eradication was carried out 4-8 weeks after treatment. Information on adherence to treatment and side effects was recorded. Results: Thirty cases were analyzed; $56.7 \%$ (17) new patients and $43.3 \%$ (13) patients with at least one failure. In $16.0 \%$ (5) there was no confirmation of eradication; an eradication rate of $72.0 \%$ (18/25) was determined, 95\% $\mathrm{Cl} 50.6-87.9$; being $78.5 \%(11 / 14)$ in new patients versus $63.6 \%$ (7/11) in previous failures, $95 \% \mathrm{Cl}-9.6$ $54.0, p=0.318$. Discussion: The eradication rate in this group of patients was not satisfactory. Currently, treatment with levofloxacin is recommended as second-line or rescue therapy in regions with low or high resistance to clarithromycin, although resistance to quinolones has increased in recent years in several countries.

Keywords: Disease eradication, Microbial Drug Resistance, Helicobacter pylori, Levofloxacin. 